\title{
Grid congestion mitigation and battery degradation minimisation using model predictive control in PV-based microgrid
}

\author{
Unnikrishnan Raveendran Nair * Monika Sandelic, Ariya Sangwongwanich, \\ Tomislav Dragičević, ${ }^{\dagger}$ Ramon Costa-Castelló and Frede Blaabjerg ${ }^{\ddagger}$
}

October 28,2020

\begin{abstract}
Increasing integration of photovoltaic (PV) system in electric grids cause congestion during peak power feed-in. Battery storage in PV systems increases self-consumption, for consumer's benefit. However with conventional maximising self consumption (MSC) control for battery scheduling, the issue of grid congestion is not addressed. The batteries tend to be fully charged early in the day and peak power is still fed-in to grid. This also increases battery degradation due to increased dwell time at high state of charge (SOC) levels. To address this issue, this work uses a model predictive control (MPC) for scheduling in PV system with battery storage to achieve multiple objectives of minimising battery degradation, grid congestion, while maximising self consumption. In order to demonstrate the improvement, this work compares the performances of MPC and MSC schemes when used in battery scheduling. The improvement is quantified through performance indices like self consumption ratio, peak power reduction and battery capacity fade for one-year operation. An analysis on computation burden and maximum deterioration in MPC performance under prediction error is also carried out. It is concluded that, compared to MSC, MPC achieves similar self consumption in PV systems while also reducing grid congestion and battery degradation.
\end{abstract}

Keywords: Model predictive control, energy management, grid congestion, PV system, battery storage, degradation.

\section{Introduction}

The last decade has seen more than doubling of renewable generation capacity, mainly driven by photovoltaic (PV) and wind power systems integrated in power grids [1]. This increased penetration of load-decoupled intermittent renewable sources has caused grid congestions, voltage regulation and stability issues [2-4] in power networks.

The negative impacts can be overcome by using energy storage systems (ESS) like batteries, which are being increasingly adopted in PV systems [6-8]. However, integration of ESS alone will not solve the issues arising from the addition of renewable sources. An effective control (energy scheduling) strategy for the battery ESS (BESS) is also essential to ensure that PV systems are grid friendly. In many European countries the extend of PV integration in the grid has resulted in a grid parity where, for a consumer it is cheaper to utilise their own PV generation than buying from the grid [9-11]. As per existing studies [7], meeting at least $65-75 \%$ of total load demand from the PV power generated at consumer's premises provide economic benefit for the consumer. This has resulted in the widely used maximising self-consumption control (MSC) in PV-BESS system [7] to maximize PV power usage by consumer. In the MSC strategy, PV power is stored in BESS as soon as surplus power is available until full charge of BESS as shown in Fig.1. Though economically beneficial, MSC may lead to grid congestion. The reason being charging BESS as soon as surplus power is available forces BESS to reach fully charged state early in the day as shown in Fig.1. Therefore when peak PV generation occurs BESS capacity cannot be used, resulting in peak power being fed-in to grid. Such power injection from many PV systems can lead to grid congestion and voltage rise at PCC if there is no sufficient load demand in the grid [12].

${ }^{*}$ U.Raveendran Nair and R.Costa-Castelló are with Institut de Robòtica i Informatica Industrial, Universitat Politècnica de Catalunya, 08034 Barcelona, Spain,(e-mail: uraveendran@iri.upc.edu, ramon.costa@upc.edu)

${ }^{\dagger}$ M.Sandelic, A.Sangwongwanich, T.Dragičević and F.Blaabjerg are with the Department of Energy Technology, Aalborg University, Aalborg 9220, Denmark.(e-mail: mon@et.aau.dk;ars@et.aau.dk; tdr@et.aau.dk ; fbl@et.aau.dk)

¥ This work is done as part of project which has received funding from the European Union's Horizon 2020 research and innovation programme under the Marie Skłodowska Curie grant agreement No 675318 (INCITE). This work is supported by the Spanish State Research Agency through the Maria de Maeztu Seal of Excellence to IRI (MDM-2016-0656). This work has been partially funded by the Spanish national project DOVELAR ref. RTI2018-096001-B-C32 (MCIU/AEI/FEDER, UE). 


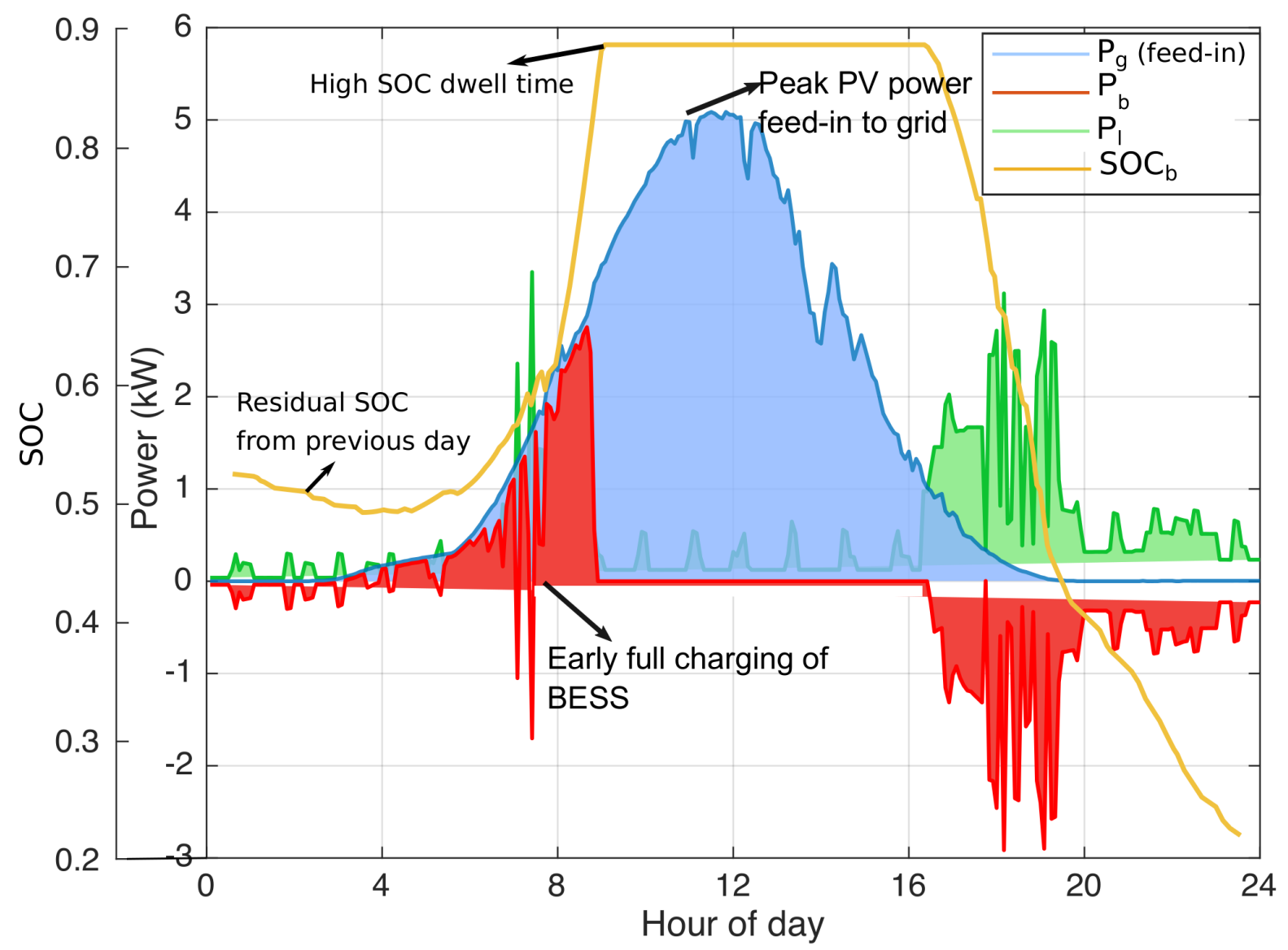

Figure 1: Typical BESS, SOC and grid feed-in profile with maximising self-consumption strategy for high PV generation scenario. Early full charging of BESS and ensuing peak PV power feed-in is shown. Profiles are based on data from [5].

Another major drawback with the MSC strategy is related to BESS degradation. The major ageing mechanisms in BESS are the calendar and cycling ageing. The calendar ageing arises in Li-ion batteries being kept at high SOC for long durations $[13,14]$ whereas cycling ageing arises from repeated deep charging/discharging cycles of BESS [15, 16]. In PV BESS system eliminating charge/discharge cycles is inevitable. However, triggering factors for calendar ageing can be reduced. In MSC strategy the early full charging of BESS results in longer dwell times at high state of charge (SOC) levels as shown in Fig.1, thereby accelerating calendar ageing. Furthermore, charging of BESS whenever surplus power is available also results in a charging behaviour without accounting for load demand. This can lead to BESS storing more charge than demanded by the load for a particular day. As a result, at the end of the day the BESS may not completely discharged leading to residual charge in it. This residual charge can be observed in Fig.1 at the beginning of the day. This residual charge also leads to increased dwell time of BESS at a charged state, further aggravating the calendar ageing. Therefore, grid congestion and battery degradation are the two major concerns when employing a MSC strategy.

Scheduling PV-BESS system aided by forecast of generation and load profiles can alleviate these drawbacks. The knowledge of generation, load profiles can be used to improve the energy scheduling and thereby reduce peak power injection and BESS degradation. These approaches have been implemented in $[9,17,18]$ where the decision on BESS scheduling was done offline based on forecast value of generation and load.

Model predictive controller (MPC) provide a solution for optimal scheduling using forecast values while allowing for an online implementation. Unlike offline solutions, MPC makes decisions based on the current system data and updated forecast values at any instance allowing for more optimal results. Though MSC can be modified to include the forecast information, this does not necessarily guarantee an optimal solution. The MSC is a rule based method, where the explicit rules determine the optimality of the solution which in turn requires prior understanding of system behaviour. The MPC on the other hand generates decision by solving an optimisation problem which always guarantee an optimal solution. Apart from this the required system behaviour can be defined implicitly though the optimisation problem without relying extensively on prior knowledge of system behaviour.

In [19-23], the MPC has been applied for optimal economic energy scheduling in grids either to achieve operating cost minimisation or energy arbitrage. In [24] the MPC was used in smoothing the output from a wind farm using a battery 


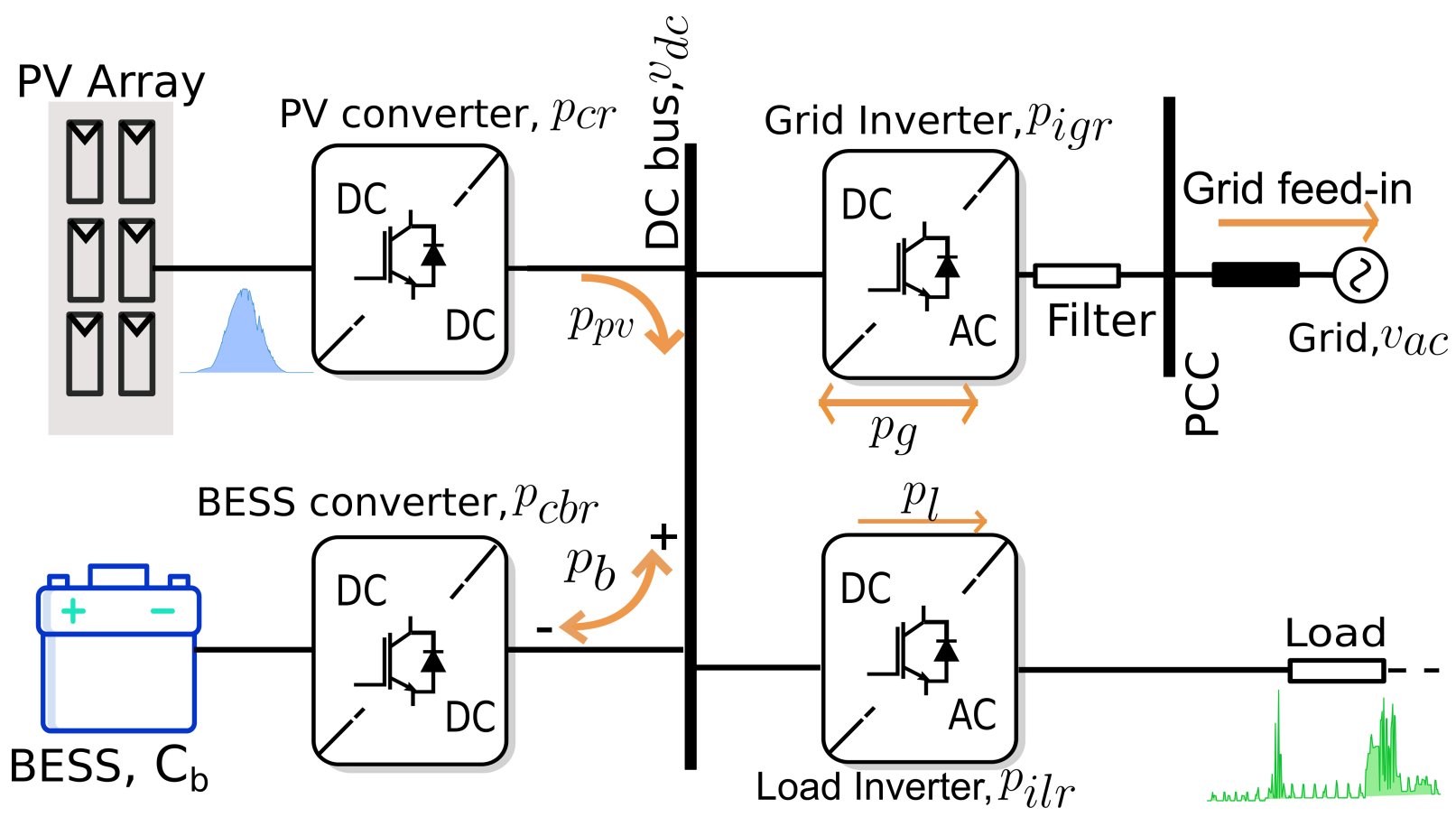

Figure 2: Schematic of the test case microgrid [5].

system. The utilisation of MPC for management of plug-in hybrid vehicle charging was demonstrated in [25]. Predominantly the application of MPC in electrical systems, based on previous works, had been limited to economic optimisation of the system operation. However, the ability of MPC can be extended beyond the above mentioned works to a PV BESS system in improving the electrical performance (grid congestion), ESS lifetime (degradation) while also maintaining economic PV BESS operation (self consumption). Such a multi objective scheduling with MPC that modifies the charging profile of BESS in a PV system have not been carried out before. The long term improvements that can be achieved with MPC have not either been quantified or demonstrated in previous works.

In this context the major contribution of this work is the application of MPC in energy scheduling of a PV BESS system to achieve the objectives of grid congestion mitigation, minimising BESS degradation and maximising self consumption. The PV BESS system is emulated using the data from a test case microgrid in Lindenberg, Germany [5]. One year's data have been used in this work. This utilisation of one year data allows for demonstrating and quantifying the long term improvements achieved with MPC in comparison to MSC strategy applied to the same system. The work also tries to quantify the maximum deterioration in performance that can arise in MPC under the prediction error of generation, load forecast, so as to highlight the worst case scenario. Finally, the work also analyses the computational requirement needed for the online implementation of MPC.

The rest of the paper is organised as follows. Section II presents the PV-BESS system considered in this work. The MPC based energy management framework is introduced in Section III along with the optimisation problem formulation for MPC. The results are discussed in Section IV and the work is concluded in Section V.

\section{System description}

The PV BESS system considered in this work is shown in Fig.2. It represents a DC coupled configuration where both the $\mathrm{PV}$ and BESS are connected to the same DC bus via interfacing DC/DC converters. The PV converter is unidirectional and works in boost mode with power flow to the grid. The BESS converter is capable of bi-directional operation allowing for compensating the imbalance power in the DC bus. The BESS converter will work in buck-boost mode. The main grid is interfaced to the system through a DC/AC converter and filter. The converters allow control of power flow from the PV, BESS and grid.

The PV profile is obtained from the irradiation and ambient temperature data measured at Lindenberg, Germany [5] and will be used as set points for the PV converter. It should be noted that, in this work, the PV system is always assumed to be working at the maximum power point (MPP). The PV converter in Fig.2 is considered to be implemented with the maximum power point tracking (MPPT) control [26] which always ensure the maximum possible power from the PV array. The energy management problem considered in this work will only deal with the set point generation of BESS converter and grid converter of Fig. 2. The aspects of MPPT control is not assessed here as it is beyond the scope of this paper. 
Table 1: Parameter values for PV BESS system.

\begin{tabular}{|c|c|}
\hline Parameter & Value \\
\hline Rated power of PV array & $6 \mathrm{~kW}$ \\
PV converter rated power, $p_{c r}$ & 6 \\
BESS capacity, $c_{b}$ & $9.375 \mathrm{kWh}$ \\
BESS converter rated power, $p_{c b r}$ & $3 \mathrm{~kW}$ \\
Load inverter rated power, $p_{i l r}$ & $3 \mathrm{~kW}$ \\
Grid interfacing inverter rated power, $p_{i g r}$ & $7 \mathrm{~kW}$ \\
DC link voltage, $v_{d c}$ & $450 \mathrm{~V}$ \\
Nominal voltage (AC), $v_{a c}$ & $230 \mathrm{~V}$ \\
\hline
\end{tabular}

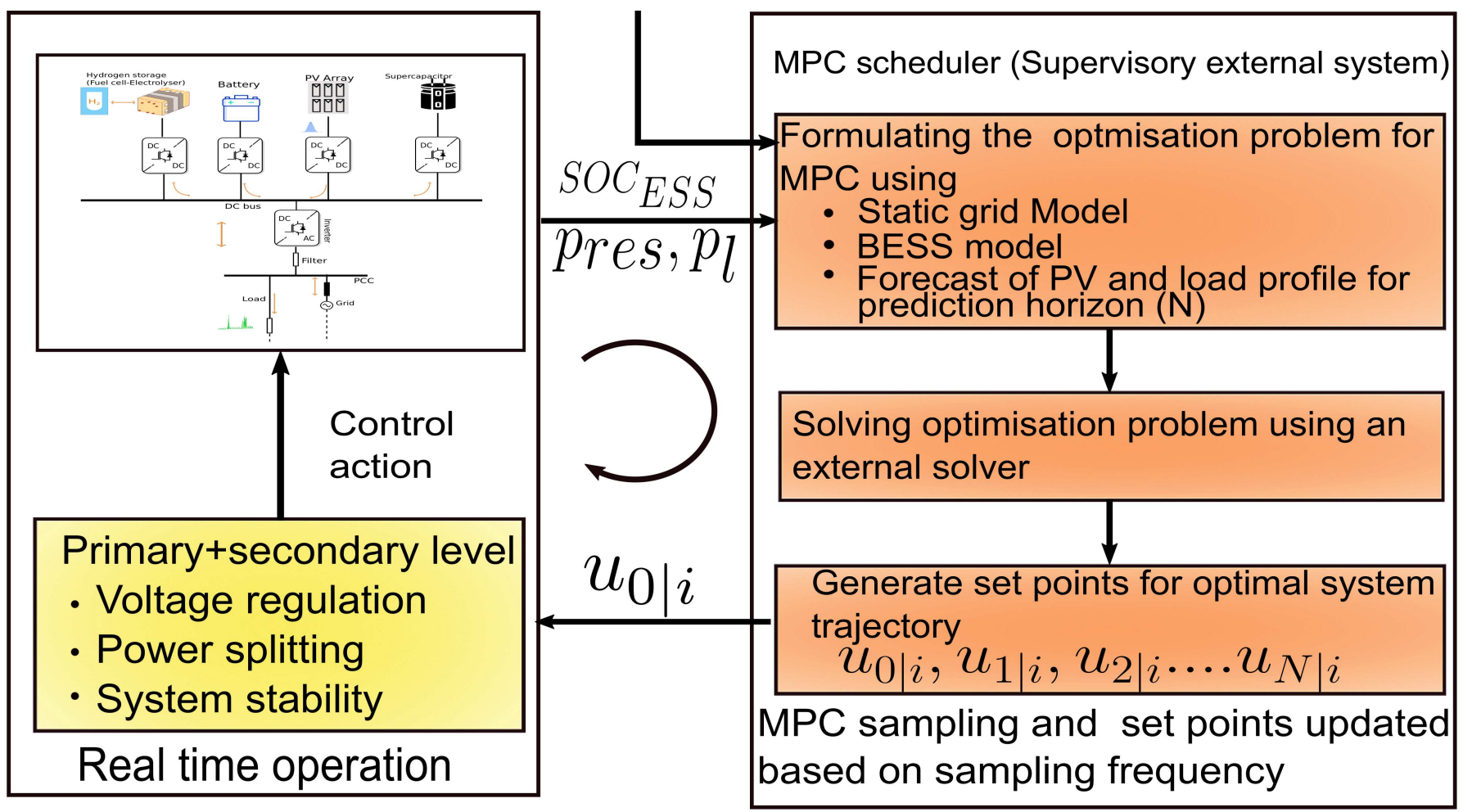

Figure 3: Flow-chart showing the MPC scheduling process in the PV BESS system.

The load emulated represents a 4-person household with an annual consumption of 4.5 MWh. As the objective of this work is on improving the self-consumption in a PV-BESS by improving the control strategy, the problem of optimal sizing of BESS is not considered in detail in this work. Nevertheless, based on the guidelines presented in $[9,12,27]$ the BESS sized such that there was $1.55 \mathrm{kWh}$ capacity per $\mathrm{kW}$ of installed PV power. The relevant parameters of the PV BESS system and converter ratings are given in Table 1.

\section{Energy Management framework with MPC}

The objective of a generic control system for the PV BESS system in Fig.2 will be to ensure a stable operation and optimal management of energy in the system. The stable system operation will be ensured by the real-time low-level controllers that ensures voltage, frequency regulation (AC side) by keeping these parameters within prescribed limits. The energy management (considered in this work) will be handled by the high-level controllers and the output from this stage will be provided to low-level control to guide them. A detailed assessment of the low-level controllers are not carried out in this work for the sake of brevity as they are beyond the scope as energy is the main focus. It should be noted that the MPPT will be implemented in the low level control of the PV converter.

In the high-level controller for energy management, an implicit receding horizon MPC is considered in this work [28]. At every sampling instant, $i$, MPC is provided with forecast of generation and load for a fixed window length, defined by $N$ points, called prediction horizon. The sampled system state, generation/load value at instant $i$ along with the available forecast will then be used by the controller to predict the system evolution for the prediction window using set points generated by 
MPC. The set points pertaining to the system trajectory that define the optimal behaviour based on a defined criteria will be selected. Therefore, for a prediction horizon of length $N$ the MPC will generate $N+1$ set points $u_{0 \mid i}, u_{1 \mid i}, u_{2 \mid i} \ldots u_{N \mid i}$ where $u_{k \mid i}=u(i+k) \forall k=0,1,2 . . N$. Among the set points generated, the first one, $u_{0 \mid i}$, will be applied to PV BESS system. The $u_{0 \mid i}$ in this case comprises of BESS $\left(p_{\mathrm{b}}\right)$ and grid $\left(p_{\mathrm{g}}\right)$ converters' power set-points. This process will be repeated at every sampling instance with the current system states, generation/load values and updated predictions [29]. Therefore, at every sampling instant the MPC makes its decision considering a prediction window shifted by one sampling time resulting in the receding nature of MPC. This decision making process with MPC is demonstrated through flow-chart in Fig.3. Analytically the process of identifying the optimal trajectory set points with MPC is done by solving a constrained optimisation problem.

In the case of application of MPC in PV BESS system the sampled system state (SOC of BESS) can be obtained using a dynamic model as shown in [30]. The set points generated from the MPC can be the input to the dynamic model. Such a dynamic modelling approach has shown to be sufficiently accurate for energy management problem [9]. Additionally, such model can easily include updates on faded capacity and evaluate the battery dynamics with the respect to the available capacity at any given state of health (SOH) of the BESS [30].

\subsection{Cost function for energy management problem}

The energy scheduling objective is to minimise grid congestion, BESS degradation while maximising the self consumption. The cost function chosen to ensure the same is

$$
J=\sum_{k=i}^{i+N}\left(J_{\mathrm{g}}(k)+J_{\mathrm{b}}(k)\right) .
$$

The first term, $J_{\mathrm{g}}$ in the above cost function is given by

$$
J_{\mathrm{g}}(k)=\lambda_{1} \cdot p_{\mathrm{g}}(k)^{2}
$$

where $\lambda_{1}$ is a weighting factor. As it can be seen, $J_{\mathrm{g}}$ penalises power exchange with the grid, the effect of which is twofold. Firstly by penalising grid power, the controller tries to reduce the peak power value that is being injected to the grid. This aims to reduce grid congestion. Secondly, this forces the PV power generated to be utilised, as much as possible, by the consumer thus promoting self-consumption.

The second term in (1) $J_{\mathrm{b}}$ is chosen as

$$
J_{\mathrm{b}}(k)=\lambda_{2} \cdot S O C_{\mathrm{b}}(k)^{2}+\lambda_{3} \cdot \Delta S O C_{\mathrm{b}}(k)^{2}
$$

where $\lambda_{2}, \lambda_{3}$ are weighting factors and $S O C_{\mathrm{b}}$ is the BESS SOC. This term deals with minimising rate of degradation of BESS. The penalisation $S O C_{\mathrm{b}}$ tries to limit high SOC values in the BESS thus limiting the calendar ageing. The $\triangle S O C_{\mathrm{b}}=S O C_{\mathrm{b}}(k+1)-S O C_{\mathrm{b}}(k)$ minimises the change in SOC thus indirectly reduces excessive charge/discharge cycle which affect the cycling ageing in BESS. The exact analytical equation that represents BESS degradation [15] is highly non-linear. An explicit utilisation of the same will result in a complex non-linear optimisation problem that can be difficult to solve [31]. In order to avoid this, the quadratic formulation in (3) has been maintained. The resulting quadratic problem, though non-linear, has very efficient algorithms for solving them and guarantees a global optimum [32].

\subsection{Constraints}

The cost functions having been defined, the system constraints are discussed next to ensure that the operating limits are not violated, SOC evolution in BESS is accounted for and power balance in the system is maintained.

\subsubsection{BESS model}

The BESS model utilised is based on the Coulomb counting approach [30] which demonstrates the $S O C_{\mathrm{b}}$ evolution based on BESS power set point $p_{\mathrm{b}}$. Considering $S O C_{\mathrm{b}}$ as the system state $x$, the BESS model is defined as

$$
x(k+1)= \begin{cases}x(k)-\frac{T_{s} \cdot \eta}{C_{\mathrm{b}}} \cdot p_{\mathrm{b}}(i) & \text { if } p_{\mathrm{b}} \leq 0 \\ x(k)-\frac{T_{s}}{\eta \cdot C_{b}} \cdot p_{b}(i) & \text { if } p_{b}>0\end{cases}
$$

where $\eta$ is the power converter efficiency and $T_{s}$ is sampling time. The above model is a hybrid representation of BESS wherein the system behaviour differs based on the nature of $p_{\mathrm{b}}$. It should be noted that typically $\eta$ for the converter system 
can vary based on the operating point $\left(p_{\mathrm{b}}\right)$ of the converter. Nevertheless, in the optimisation problem formulation a constant value is chosen for $\eta$. This is to prevent a very non linear formulation of the above constraint which can arise from defining $\varsigma ̧ \eta$ as a function of $p_{\mathrm{b}}$. The use of a constant $\eta$ can also be justified, as typical converter systems have a near equal efficiency curve from $20 \%$ of rated power output [33].

\subsubsection{Grid model}

The grid is represented as a static model using equality constraint to ensure power balance given by

$$
p_{\mathrm{b}}(k)+p_{\mathrm{g}}(k)+p_{\mathrm{pv}}(k)+p_{\mathrm{l}}(k)=0
$$

where $p_{\mathrm{pv}}, p_{\mathrm{l}}$ are PV and load power respectively. The $p_{\mathrm{pv}}$ in the above represents the MPP of the PV array at any instance.

\subsubsection{Physical operating constraints}

The physical constraint to ensure that BESS is not over-charged or deep-discharged, which increases its rate of degradation [16] can be given as

$$
x^{l o} \leq x(k) \leq x^{u p}
$$

where, $x^{l o}, x^{u p}$ are the permissible lower and upper limits of $S O C_{\mathrm{b}}$. The above represents a hard constraint on the system state which can, in some cases, lead to a non-feasible solution in the optimisation problem. In this scenario, in order to ensure that the on-line implementation of MPC is reliable the above hard constraints are replaced with soft constraints [34]. The soft constraints allow for constraint violation but at a high penalty to the cost function. This ensures that under most of the conditions the SOC operating limits are not violated. The soft constraint implementation of (6) can be given as

$$
x^{l o}-\epsilon_{b}(k) \leq x(k) \leq x^{u p}+\epsilon_{b}(k)
$$

where $\epsilon_{b}$ is the violation of the SOC bounds. In order to ensure that the violations are rare an additional term is added to the cost function (1) given by

$$
J_{\text {vio }}(k)=\lambda_{4} \cdot \epsilon_{b}^{2}(k)
$$

where $\lambda_{4}$ is weighting the $\epsilon_{b}$. Choosing a high value for $\lambda_{4}$ will ensure that $\epsilon_{b}$ value is kept minimal during the scheduling while also ensuring the feasibility of optimisation problem. In practice this can be decided through trial and error such that the violation represented by $\epsilon_{b}$ is minimised.

\subsubsection{Electrical operating constraints}

The electrical constraints ensure that operating points of DC/DC converters are kept within their rated values. This is given by

$$
\begin{aligned}
-p_{\text {cbr }} & \leq p_{\mathrm{b}}(k) \leq p_{\text {cbr }} \\
-p_{\text {igr }} & \leq p_{\mathrm{g}}(k) \leq p_{\text {igr }}
\end{aligned}
$$

where $p_{\mathrm{cbr}}, p_{\mathrm{igr}}$ are as shown in Table 1.

\subsubsection{Mixed Logical Dynamics formulation}

The hybrid BESS model of (4) cannot be directly utilised in the optimisation problem. In order to use them they need to be transformed into a mixed logical dynamic (MLD) model, that provides a linear equality representation of (4). The guidelines in [35] is used in this transformation to MLD. In (4) the value of $p_{\mathrm{b}}$ defines the BESS behaviour. A boolean variable $\delta_{b}$ is now introduced to define the nature of $p_{\mathrm{b}}$ such that when $\left[\delta_{b}(i)=1\right] \leftrightarrow\left[p_{b}(i) \geq 0\right]$. This MLD representation of this condition is given by

$$
\begin{gathered}
p_{c b r} \cdot \delta_{b}(k) \leq p_{\mathrm{b}}(k)+p_{c b r} \\
-p_{c b r} \cdot \delta_{b}(k) \leq-p_{\mathrm{b}}(k) .
\end{gathered}
$$


This introduction of boolean variable allows (4) to be represented as

$$
x(k+1)=x(k)+\frac{T_{s}}{C_{b}} \cdot \delta_{b}(i) \cdot p_{\mathrm{b}}(i) \cdot\left(\eta-\frac{1}{\eta}\right)-\frac{T_{s} \cdot \eta}{C_{b}} \cdot p_{b}(k)
$$

The multiplicative term above is eliminated using an auxiliary variable $z_{b}(i)=\delta_{b}(i) \cdot p_{\mathrm{b}}(i)$ to maintain a linear formulation of (11). This multiplication is realised in MLD domain as

$$
\begin{aligned}
& z_{b}(k) \leq p_{c b r} \cdot \delta_{b}(k) \\
& z_{b}(k) \geq-p_{c b r} \cdot \delta_{b}(k) \\
& z_{b}(k) \leq p_{\mathrm{b}}(k)+p_{c b r} \cdot\left(1-\delta_{b}(k)\right) \\
& z_{b}(k) \geq p_{\mathrm{b}}(k)+-p_{c b r} \cdot\left(1-\delta_{b}(k)\right)
\end{aligned}
$$

resulting in (4) represented as

$$
x(k+1)=x(k)+\frac{T_{s}}{C_{b}} \cdot \delta_{b}(k) \cdot\left(\eta-\frac{1}{\eta}\right)-\frac{T_{s} \cdot \eta}{C_{b}} \cdot p_{b}(k)
$$

The cost functions, constraints having been defined, the resulting optimisation problem considered in MPC based scheduling in PV-BESS system is summarised by

$$
\min _{p_{\mathrm{b}}, p \mathrm{~g}}\left[J\left(p_{\mathrm{b}}, p \mathrm{~g}, x\right) \triangleq \sum_{k=i}^{k+N}\left[J_{b}(k)+J_{\mathrm{g}}(k)+J_{\mathrm{vio}}(k)\right]\right]
$$

subject to

$$
\text { ESS model (13) in } x
$$

Grid model (5)

Constraints (7),(10), (12)

\section{Results}

The optimisation problem in MPC is a mixed integer quadratic problem (MIQP) which is solved with Branch and bound algorithms using Gurobi [36] as solver in MATLAB. The MPC was run in an Intel i7 2 core, $2.5 \mathrm{GHz}$ machine with $8 \mathrm{~GB}$ RAM. The data used from Lindenberg, Germany [5] which emulated load and generation was sampled at 5 min.

\subsection{Performance indices}

Prior to presenting the results some performance indices are introduced which allows for quantifying the PV BESS system behaviour achieved with energy scheduling. The self consumption is quantified using the annual self-consumption ratio (ASCR) defined as the following

$$
A S C R=\frac{p v_{\text {cons }}}{p v_{\text {gen }}} \cdot 100(\%)
$$

where $p v_{\text {cons }}$ is the amount of PV energy generated that has been utilised by the consumer through the load demand and BESS storage, while $p v_{g e n}$ is the total annual PV energy generated. In order to ensure maximum economic benefit for the consumer, this value should be as high as possible.

The BESS end-of-life is defined when the capacity has faded to $80 \%$ of the nominal rated value $\left(C_{\mathrm{b}}\right)$. Under this scenario, the effect of scheduling on BESS degradation is quantified by assessing the capacity loss in the battery after one year's scheduling. The degradation model of Li-ion battery provided in [15] will be used. The BESS SOC profile from the different scheduling methods will be used to calculate annual capacity fade $\left(C_{f}\right)$ of BESS due to its utilisation. The entire process in the calculation of $C_{f}$ from BESS SOC profile $\left(S O C_{b}\right)$ is represented using the flow chart shown in Fig. 4 where $T_{a}$ is ambient temperature, also available in the Lindenberg data.

Finally, the electrical performance associated with grid congestion will be assessed through the peak power injected into the grid using the index, annual average peak power reduction (APPR). This index is defined as

$$
A P P R=\frac{1}{D} \sum_{n=1}^{D}\left(\frac{p_{\mathrm{pvp}(\mathrm{n})}-p_{\mathrm{gp}(\mathrm{n})}}{p_{\mathrm{pvp}(\mathrm{n})}}\right) \cdot 100(\%)
$$




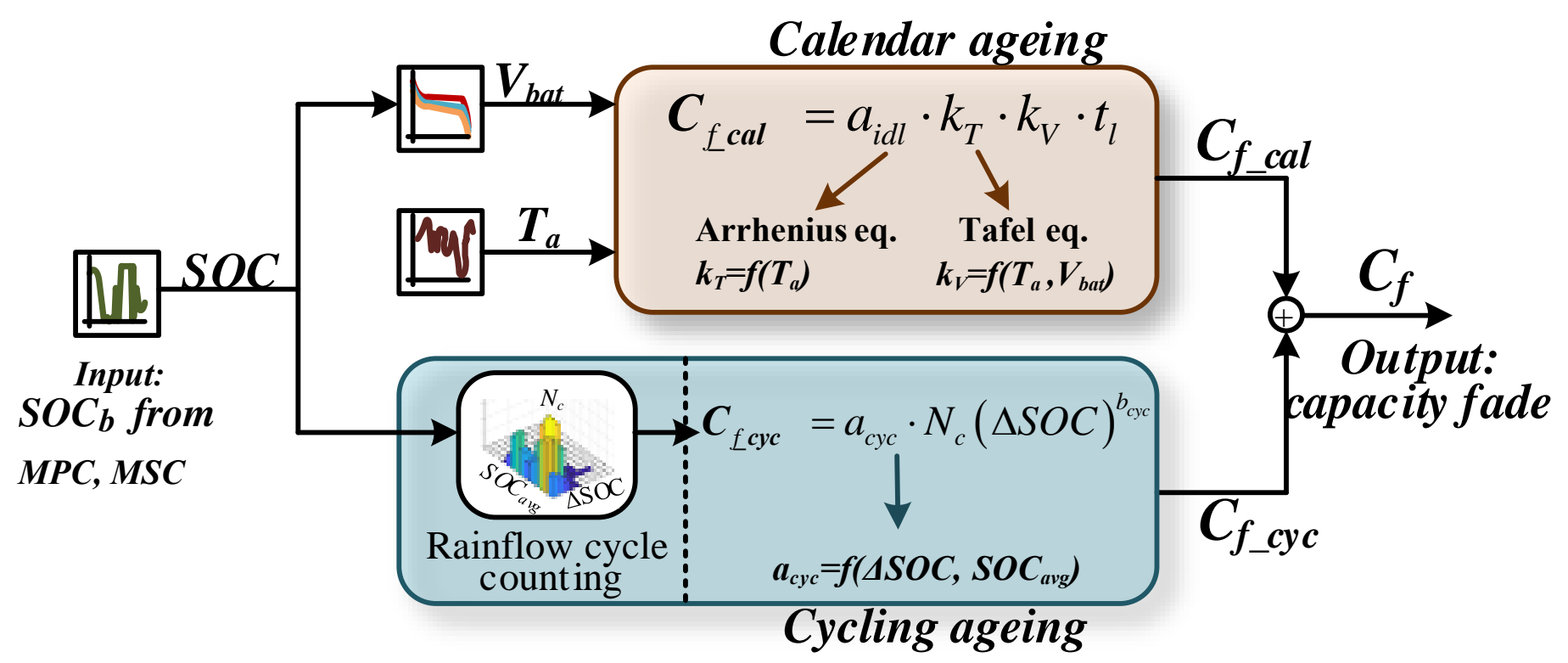

Figure 4: Flow chart for capacity fade, $C_{f}$, mapping from $S O C_{b}$ with overview of calendar and cycling ageing calculation.

Table 2: Parameter values used in MPC.

\begin{tabular}{|l|l|}
\hline Parameter & value \\
\hline Sampling time $\left(T_{s}\right)$ & $5 \mathrm{~min}$ \\
$\mathrm{~N}$ & 288 \\
$x^{l o}$ & 0.1 \\
$x^{u p}$ & 0.9 \\
$\lambda_{1}, \lambda_{2}, \lambda_{3}$ & $500,400,3$ \\
$\lambda_{4}$ & 1000 \\
$\rho$ & 100 \\
$\mathrm{D}$ & 365 \\
$\eta$ & 0.95 \\
\hline
\end{tabular}

where $D$ is the total of days in a year, $p_{\text {pvp }}(n)$ is the peak PV power injected to grid for the $n^{\text {th }}$ day without BESS integration, and $p_{\mathrm{gp}(\mathrm{n})}$ the peak grid power on the $n^{\text {th }}$ day with BESS integration under a scheduling strategy (MSC or MPC). This index gives the percentage reduction in peak power achieved with a scheduling method in comparison to the case where the BESS integration was not available. The parameters for MPC based BESS scheduling is given in Table 2. The prediction horizon value, $\mathrm{N}$, was chosen to be 24 hours considering the daily periodicity of the PV and load profiles.

\subsection{Analysis of the scheduling results with MPC}

The results for the annual scheduling in the PV BESS system achieved with MSC and MPC is shown in Fig. 5a, Fig.5b respectively. The results presented in this section considers the deterministic case where it is assumed that the system behaviour follows the predicted generation and load profile. The improvement in scheduling performance achieved with MPC can be better conceived through Fig. 6 which shows the BESS charging profile for the same day as in Fig. 1. The MPC scheduling with its knowledge of future generation and load profile shifts the BESS charging to the period of peak generation. This allows MPC to minimise the cost function of (14) and achieve the desired performance criteria. This shifting results in BESS being fully charged later in the day leading to lower dwell times at high SOC levels which is also evident from Fig. 6. Another important observation in MPC scheduling is the absence of residual charge in BESS at the beginning of the day which is seen in the MSC scheduling shown in Fig.1. During high PV generation months (summer), the load demand may not be high enough to fully utilise the stored energy in the BESS in a day. In MSC scheduling, this will result in BESS being kept at high SOC at the end of the day. In MPC due to the penalising of the SOC value in (3) the controller is forced to reduce the BESS SOC levels. The MPC achieves this by slowly discharging the BESS back to the grid in the night (from 0 hours- 4hours), when load demand is low, in a gradual manner as shown in Fig. 6. The level to which the MPC discharges the BESS depends on the availability of PV power next day in order to ensure maximum self consumption. The elimination of residual charge with MPC is demonstrated in Fig.7 where the $S O C_{b}$ cycling with the different scheduling schemes are shown. During the high PV generation months (April- August), it can be seen that BESS is kept in a persistently charged state in the MSC scheme in comparison to the MPC. 


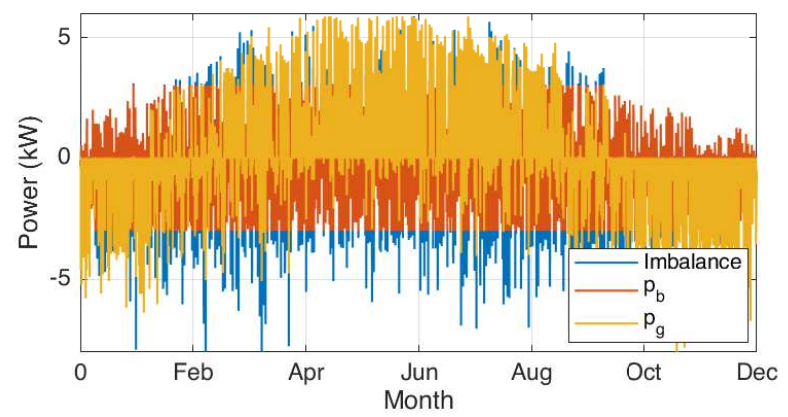

(a)

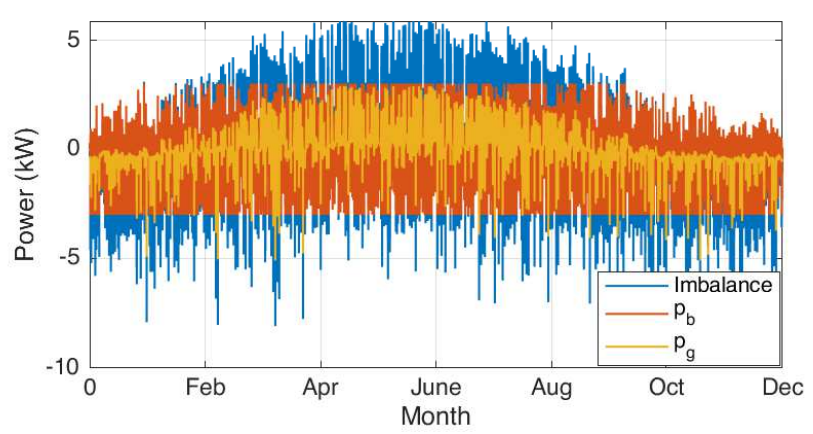

(b)

Figure 5: Scheduling results with (a) MSC, (b) MPC strategy for the PV BESS systems.



Figure 6: Battery energy scheduling with MPC for the same day as in Fig. 1.

The shifting of BESS charging to the peak generation period provides the advantage that BESS capacity is available to handle the peak power. This allows for significant reduction in the peak PV power being injected into the grid with the MPC scheme, thus promoting grid congestion mitigation. Fig. 8 shows the grid power feed-in profile with MPC and MSC for the whole year. The elimination of peak power injection with MPC is clearly observable here. In comparison to the MSC, the power injection to grid with MPC is more uniform, distributed over a larger time window and exhibits a smoother profile. Fig. 9 shows bar plot highlighting the dwell times at various power levels of interaction with the grid. It highlights that with MPC the peak power injection to grid is limited to be within $3 \mathrm{~kW}$ in comparison to the MSC scheme. The improvement in peak power injection is quantified using APPR as given in Table. 3. Comparing to PV system without any BESS integration, the MPC was capable of reducing the peak power injection by $80.38 \%$ while MSC was able to achieve only $49.72 \%$ reduction. This highlights the significant improvement in grid congestion mitigation that can be achieved by using MPC. It should also be noticed that the MPC also reduces the peak power drawn in from the grid $(\leq-5 \mathrm{~kW})$. This can be explained using Fig.8a. It can be noticed in the figure that in the winter months when the irradiation is low the MPC still stores energy in the BESS. Since the PV generation is low in these months, the BESS capacity is underutilised during this period. The MPC then uses the BESS capacity to charge from the grid during low load demand period and then use it 
MPC

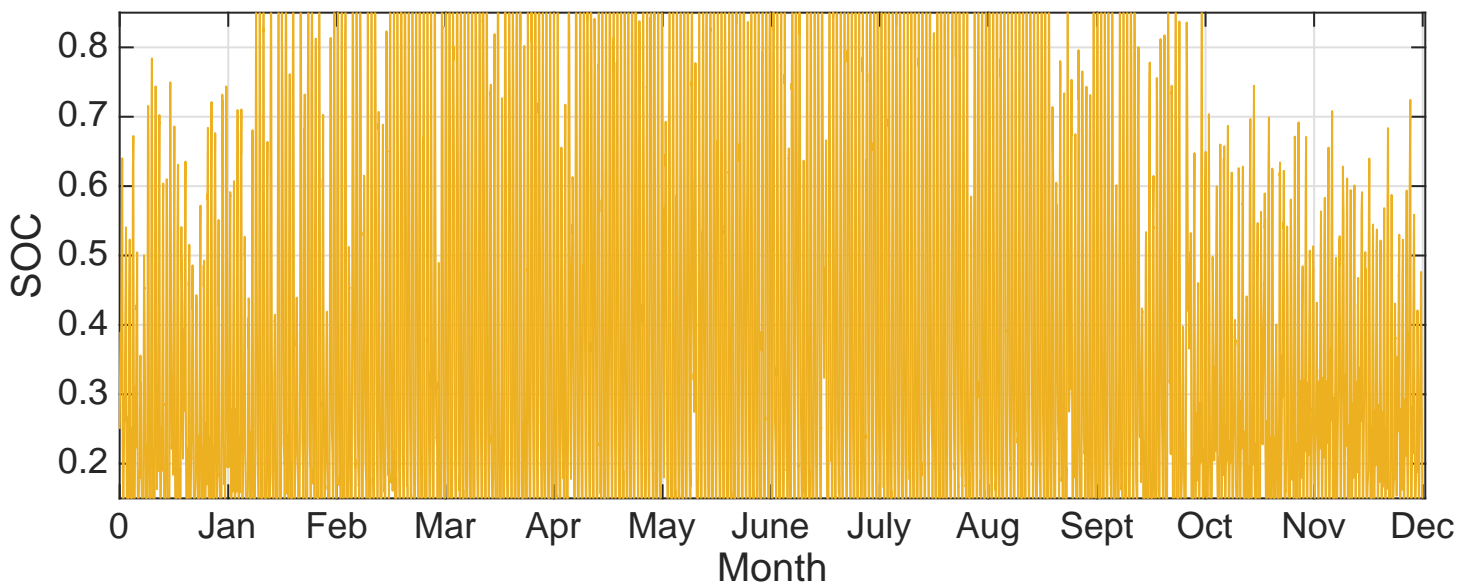

(a)



(b)

Figure 7: Annual BESS SOC cycling under (a) MPC, (b) maximum self consumption strategy.

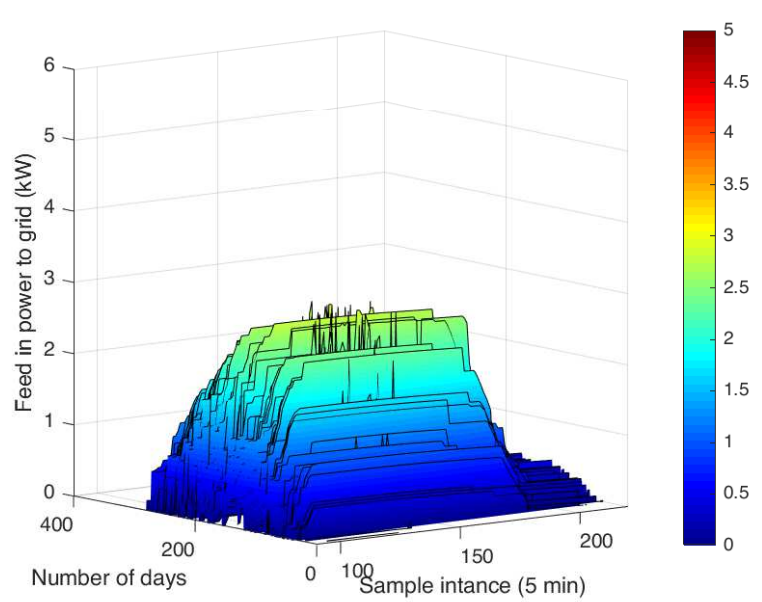

(a)

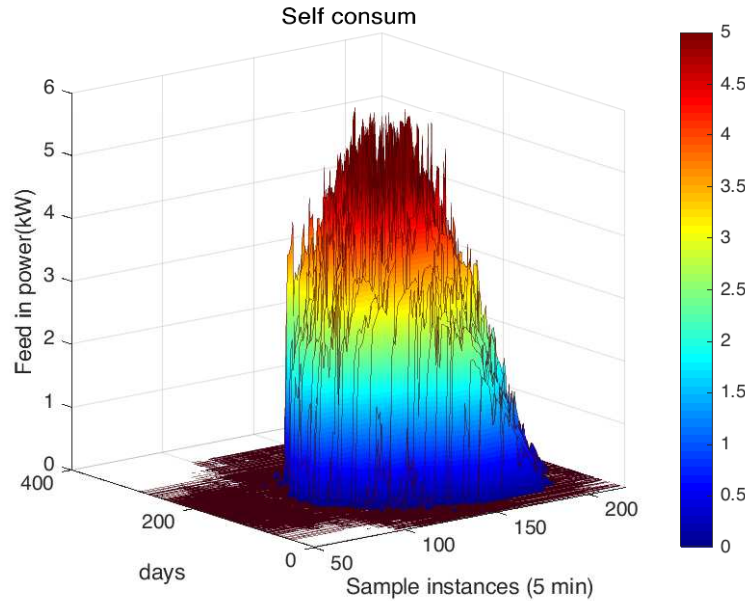

(b)

Figure 8: Annual daily grid feed-in power with (a) MPC, (b) maximum self consumption strategy

during high load demand period in the evening. This enables a reduction in annual peak power drawn-in from the grid.

It should be considered that the reduction in peak power injection with MPC can result in the grid inverter (Fig.2) operating at lower power levels than the rated power. Typically converters achieve higher efficiencies at around $20 \%$ rated power [33]. 


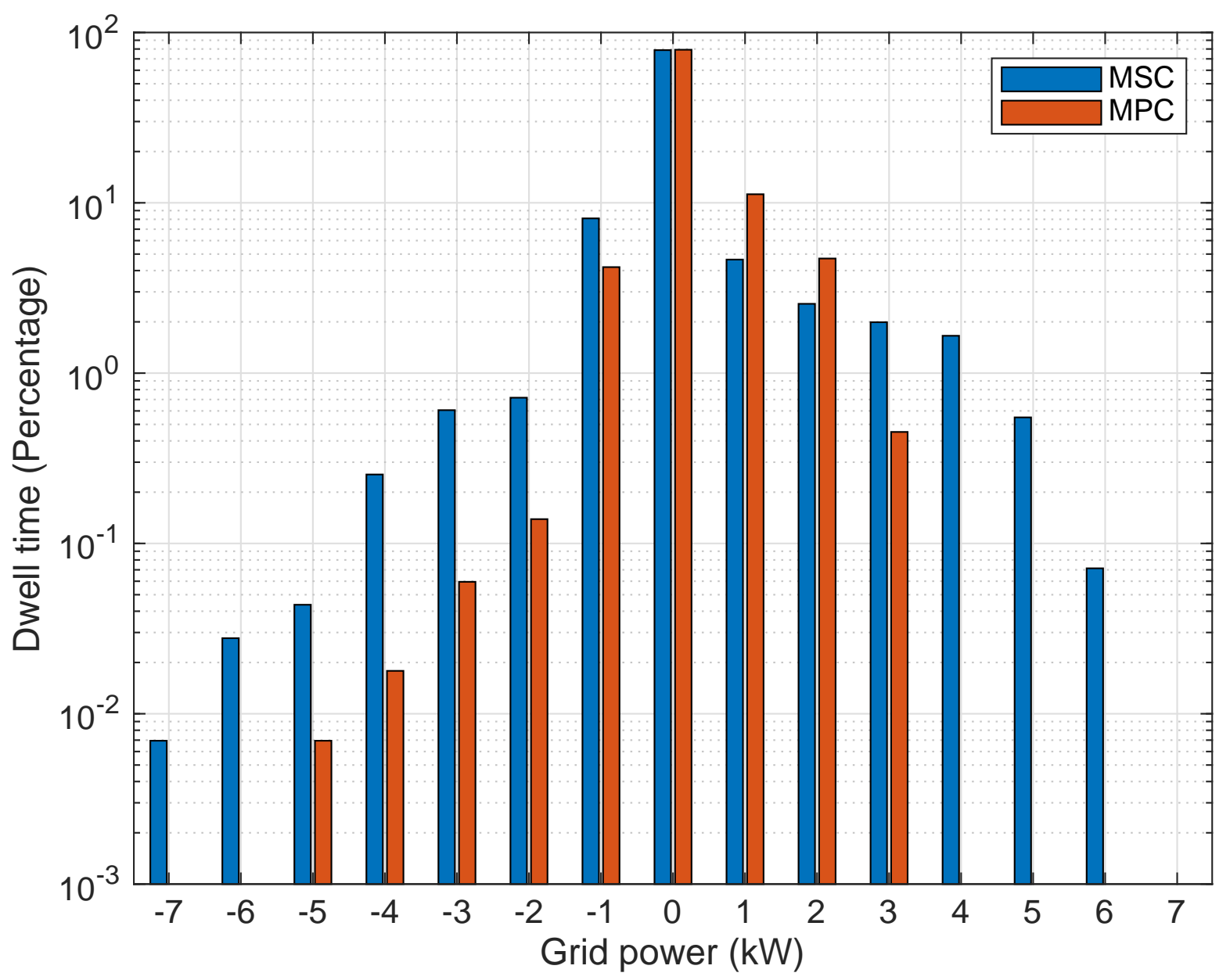

Figure 9: Bar-plot comparing the dwell times of the grid power exchanged with MPC and maximum self consumption method.

However, at very low power levels the efficiency tends to be lower. In Fig.9 it can be seen that MPC has higher dwell time for the grid inverter at low power level $(\leq 1 \mathrm{kw})$ compared to MSC. Though the efficiency at these power levels are lower, the absolute losses for the grid inverter operation is also lower since the converter is operating at a lower power point. Also, from the perspective of entire PV BESS system this does not result in overall economic loss for consumer, as PV energy yield is still maintained at the MPP and the excess power is stored in the BESS instead of being delivered to grid.

Regarding the peak power injection with MSC, it can also be considered that increasing the BESS capacity or curtailment of power injected to the grid can improve the MSC performance. However, it should be noted that both methods have their drawbacks. The high storage costs associated with BESS [37] will result in a significantly higher initial investment for the consumer when BESS capacity is increased. Also increased sizing of BESS without accounting for average load demand of consumer can be detrimental to BESS lifetime. In scenarios where the average load demand is lesser than average PV power generated, a higher capacity of BESS can lead to BESS being kept in a persistently charged state [9]. This can lead to increased calender ageing. In the case of power curtailment, limiting the power injection to grid will lead to lesser utilisation of PV power generated and economic benefit for the PV array owner. The MPC, on the contrary, demonstrated improved performance in PV BESS system without the need for increased BESS capacity or power curtailment.

Table 3: Comparison of performance indices values for different scheduling schemes

\begin{tabular}{|l||l|l|l|}
\hline Method & $\begin{array}{l}\text { ASCR } \\
(\%):\end{array}$ & $\begin{array}{l}\text { Annual } C_{f} \\
(\%):\end{array}$ & $\begin{array}{l}\text { APPR } \\
(\%):\end{array}$ \\
\hline MSC & 54.60 & 3.94 & 49.72 \\
MPC & 54.32 & 3.71 & 80.38 \\
$\begin{array}{l}\text { MPC } \\
\text { (worst- } \\
\text { case) }\end{array}$ & 52.73 & 3.65 & 72.96 \\
\hline
\end{tabular}




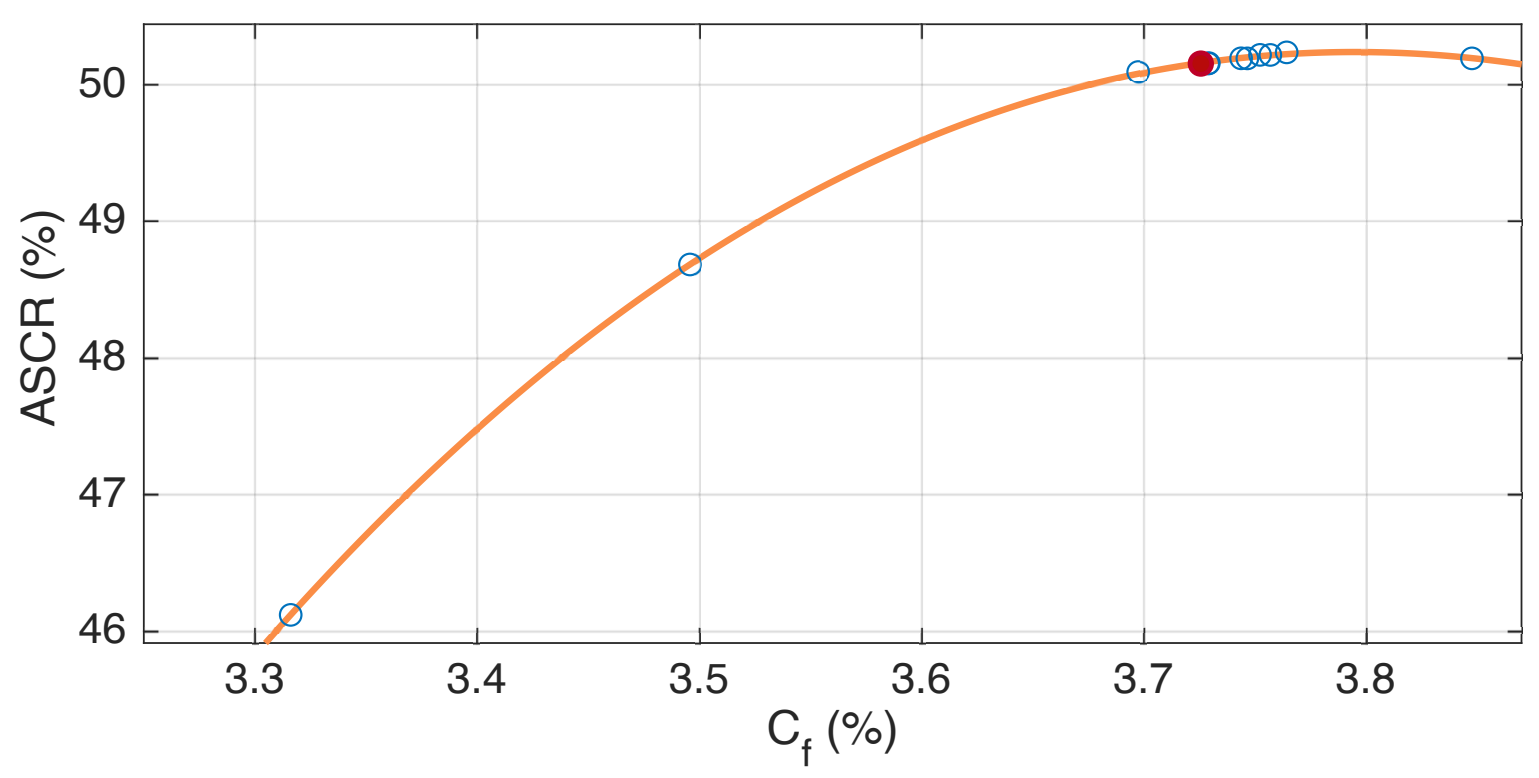

Figure 10: Variation of annual self-consumption rate with $C_{f}$ for different weighting sets of $\left(\lambda_{1}, \lambda_{2}, \lambda_{3}, \lambda_{4}\right)$. The highlighted point (red) corresponds to the value shown in Table 2.

The annual BESS degradation with the MPC and MSC is given in Table 3. As expected with the shifting of the BESS charging to peak generation period and the elimination of high SOC dwell times as shown in Fig. 5 the capacity fade with MPC is lesser than that of the MSC. The $C_{f}$ with MPC at $3.71 \%$ is a $6 \%$ reduction in comparison to the MSC.

Finally the economic performance is assessed through the self-consumption given by the annual ASCR in Table 3 . It can be seen that with MPC, the ASCR is lower than the MSC consumption case. The ASCR value for MPC at $54.32 \%$ is $99.5 \%$ compared to MSC. This slight drop arises due to multi-objective optimisation which is considered in MPC. The ASCR, $C_{f}$ exhibits complementary behaviour resulting in deterioration of one performance with the improvement in another. This can best be demonstrated through Fig.10, showing the variation of ASCR and $C_{f}$ with MPC when subjected to a parameter sweep in weighting set $\left(\lambda_{1}, \lambda_{2}, \lambda_{3}, \lambda_{4}\right)$ values (only high ASCR scenarios shown in Fig.10). It can be seen that when the ASCR is high, $C_{f}$ tends to be higher leading to increase in degradation of BESS and vice versa. The performance for the weighting set values, $\lambda_{1}, \lambda_{2}, \lambda_{3}, \lambda_{4}$, given in Table 2 is highlighted in Fig. 10 (red point). It represents an optimal trade-off between ASCR and $C_{f}$ as can be seen from results. It should also be noted that even though there is a $0.5 \%$ drop in ASCR for MPC, in comparison to MSC, the performance improvements in terms of APPR and $C_{f}$ are significantly higher in MPC scheme.

It should be noted that the determination of the weighting set values, $\lambda_{1}, \lambda_{2}, \lambda_{3}, \lambda_{4}$, as mentioned above through a random parameter sweep may not result in the identification of the most optimal weights. This can be only identified through more exhaustive methods like Pareto optimisation [38]. However, the problem of optimal weighting set determination in a multi-objective optimisation problem is not the main scope of this work and is not assessed in detail here. The approximate optimal weighting determination as discussed above facilitates the demonstration of the improvement achieved with MPC.

\subsection{Analysis of MPC performance under worst case scenario}

The results discussed so far presents MPC scheduling where actual generation and load profiles were used in prediction window (deterministic system). This allows MPC to have complete knowledge about the future generation, load demand and make decision accordingly. In the practical case forecast is bound to deviate from the predicted values. Under this scenario the performance with MPC can deteriorate. Corrective measures like stochastic MPC with chance constraining or constraint tightening [29] can improve the performance. The worst case performance arises when there are no corrective measures in place. This worst case performance is analysed in this work to quantify the maximum deterioration in performance. In order to do this, instead of actual values of generation, load demand the predicted values from a forecasting unit is used in MPC. The predictions were generated using a Neural Network (NN) trained with actual data from Lindenberg [5]. A detailed discussion on forecasting with $\mathrm{NN}$ is not provided here for the sake of brevity and considering the scope of the work. The forecast being available, at any instant $i, u_{0} \mid i$ will be sampled system values and $u_{1}\left|i, u_{2}\right| i \ldots u_{N} \mid i$ will be forecast values. The error in forecast will not be corrected and annual scheduling performance with MPC is assessed though indices value given in Table 3.

In the worst case scenario the ASCR value came down to $52.73 \%$ which is $96.5 \%$ of the MSC scheme. This $3.5 \%$ drop will 
Table 4: Analysis of computation time with varying lengths of prediction horizon.

\begin{tabular}{|l|c|c|}
\hline $\begin{array}{l}\text { Prediction } \\
\text { horizon } \\
\text { length }\end{array}$ & Average time $(\mathrm{s})$ & Worst case time $(\mathrm{s})$ \\
\hline 3 hours & 0.0146 & 0.3413 \\
6 hours & 0.0246 & 0.1932 \\
12 hours & 0.0549 & 0.4493 \\
24 hours & 0.1096 & 1.2513 \\
\hline
\end{tabular}

be the maximum possible reduction in ASCR and can be considered as a lower bound for the ASCR value. Therefore, when formulating MPC accounting for the stochastic nature of the load, generation prediction errors, it can be ensured that the ASCR value will lie between the worst and deterministic case value. The reduction in ASCR arises, as in some days the forecast of PV generation will be higher than actual value. Under this scenario, as the MPC expects more PV generation it reserves BESS capacity without charging it earlier in the day which leads to lesser self consumption. Formulating the MPC accounting for the stochastic behaviour of generation, load profiles will ensure improved performance in the ASCR. In terms of BESS $C_{f}$ the worst case performance is better than ideal MPC and MSC scheme. This arises as the energy handled by BESS in worst case scenario is lesser, as evident from the lower ASCR values. This leads to lower dwell times at charged levels in BESS causing a reduction in calendar ageing. Finally, the APPR in worst case MPC (72.96\%) is still higher than the MSC scheme (49.72\%) implying that the grid congestion mitigation with MPC even in the worst case scenario is superior to the MSC scheme. Therefore, to conclude the MPC even in worst case scenario is capable of delivering improved performance in multiple objectives (APPR and $C_{f}$ ) over the MSC without excessively deteriorating self consumption.

\subsection{Computational time analysis}

The prediction horizon of 24 hours was considered in the MPC considering daily periodicity. The MPC in comparison to the MSC can be computationally intensive. In the 24 hour prediction horizon the number of decision variables for which the optimisation problem in MPC is solved is $3 \cdot 288=864\left(p_{b}, p_{b}, S O C_{b}\right)$. In comparison the MSC always have to solve for 2 decision variables. Therefore an analysis based on computational time complexity is necessary with MPC to ensure the feasibility of an online implementation of the scheme.

The MIQP optimisation problem considered in this work is NP complete [39]. The upper bound for computation time of branch and bound algorithm, used in solving MIQP, is $\mathcal{O}\left(2^{j \cdot N}\right)$, where $j$ is the number of boolean variables in the MIQP. In this work $j=1$. This highlights that as the number of decision variables increases the computational time involved can reach very high values. However, solvers like Gurobi use techniques like presolving, cutting planes, heuristics, and parallelism that significantly improve the capabilities of the algorithms [36]. Nevertheless, it is necessary to analyse the average and the worst case computational time when solving MIQP. This is given in Table 4 where the computation times for varying lengths of prediction horizons are given. These times were obtained after doing an annual scheduling for PV-BESS system using the different prediction horizon lengths. The worst case computational time occurs in the 24 hour window and is very low (1.2513 s) compared to a 5 min sampling time. This ensures that for a PV BESS considered in this work, the MPC scheduling is affordable for online implementation with 24 hour prediction window.

It can be seen from Table 4 the average computation time with smaller prediction horizons were less. Nevertheless, the 24 hour case was considered in this work as it was giving best performance in terms of ASCR. The smaller horizons resulted in lower ASCR values as the scheduler had lesser information of future generation, load profiles.

\section{Conclusion}

The MPC scheduling was developed for the PV BESS system to overcome the issues with grid congestion and BESS degradation that arises using the MSC strategy. An MIQP optimisation problem was developed for the MPC based scheduling. A performance comparison between the MPC and MSC scheme was carried out, when used in annual scheduling of a PV BESS system, using various performance indices. The MPC demonstrated significant reduction of the peak power injection to grid (grid congestion) and capacity fade in BESS. This was achieved as MPC caused shifting of BESS charging to peak generation period thus reducing the dwell times at charged levels and power injection to grid. In terms of self consumption, the MPC achieved a similar performance compared to MSC scheme were the ASCR reduced by only $0.6 \%$. These results emphasised the capability of MPC to achieve improved performance over the MSC in multiple objectives, while achieving similar self-consumption. 
The MPC, being a scheduling method that uses generation and load forecast in its decision making, the work also tried to quantify the maximum performance deterioration that can arise during forecast errors. The analysis of the same demonstrated that under forecast errors, the ASCR with MPC will only be reduced by $3.5 \%$ compared to the MSC scheme while APPR and $C_{f}$ of BESS was better than MSC scheme Finally the work also confirmed the feasibility of practical online implementation of MPC through computational time analysis.

In terms of future work, focus can be on optimum weight determination for multi-objective optimisation problem in MPC. Apart from this, investigation can be done on stochastic MPC to address the issue of prediction errors in forecast and experimental validations.

\section{References}

[1] D. Gibb, H. Murdock, F. Appavou, A. Brown, B. Epp, B. Kondev, A. McCrone, L. Renalder, E. Musolino, J. Sawin, K. Seyboth, J. Skeen, and F. Sverrisson, "Renewables 2019 global status report," Paris: REN21 Secretariat, 2019.

[2] D. Maxwell, "Parts of Northern Ireland's electricity grid overloaded," https://www.bbc.com/news/uk-northern-ireland-24921411.

[3] B. Bayer, P. Matschoss, H. Thomas, and A. Marian, "The German experience with integrating photovoltaic systems into the low-voltage grids," Renewable Energy, vol. 119, pp. 129-141, 2018.

[4] H. Schermeyer, M. Studer, M. Ruppert, and W. Fichtner, "Understanding distribution grid congestion caused by electricity generation from renewables," in Smart Energy Research. At the Crossroads of Engineering, Economics, and Computer Science. Springer, 2017, pp. 78-89.

[5] M. Bost, B. Hirschl, and A. Aretz, Effekte von Eigenverbrauch und Netzparität bei der Photovoltaik. Institut für ökologische Wirtschaftsforschung (IÖW) GmbH, gemeinnützig, 2011.

[6] Y. Li, W. Gao, and Y. Ruan, "Performance investigation of grid-connected residential pv-battery system focusing on enhancing self-consumption and peak shaving in Kyushu, Japan," Renewable energy, vol. 127, pp. 514-523, 2018.

[7] V. Bertsch, J. Geldermann, and T. Lühn, "What drives the profitability of household pv investments, self-consumption and self-sufficiency?" Applied Energy, vol. 204, pp. 1-15, 2017.

[8] G. d. O. e Silva and P. Hendrick, "Photovoltaic self-sufficiency of Belgian households using lithium-ion batteries, and its impact on the grid," Applied Energy, vol. 195, pp. 786-799, 2017.

[9] J. Moshövel, K.-P. Kairies, D. Magnor, M. Leuthold, M. Bost, S. Gährs, E. Szczechowicz, M. Cramer, and D. U. Sauer, "Analysis of the maximal possible grid relief from pv-peak-power impacts by using storage systems for increased self-consumption," Applied Energy, vol. 137, pp. 567-575, 2015.

[10] H. Wirth and K. Schneider, "Aktuelle fakten zur photovoltaik in deutschland."

[11] C. Breyer and A. Gerlach, "Global overview on grid-parity," Progress in photovoltaics: Research and Applications, vol. 21, no. 1, pp. 121-136, 2013.

[12] M. Resch, B. Ramadhani, J. Bühler, and A. Sumper, "Comparison of control strategies of residential pv storage systems," in Proc 9th International Renewable Energy Storage Conference and Exhibition (IRES 2015), Messe Düsseldorf, 9-11 March 2015, 2015, pp. 1-18.

[13] J. Schmalstieg, S. Käbitz, M. Ecker, and D. U. Sauer, "A holistic aging model for li (nimnco) o2 based 18650 lithium-ion batteries," Journal of Power Sources, vol. 257, pp. 325-334, 2014.

[14] D.-I. Stroe, M. Świerczyński, A.-I. Stan, R. Teodorescu, and S. J. Andreasen, "Accelerated lifetime testing methodology for lifetime estimation of lithium-ion batteries used in augmented wind power plants," IEEE Transactions on Industry Applications, vol. 50, no. 6, pp. 4006-4017, 2014.

[15] G. Angenendt, S. Zurmühlen, H. Axelsen, and D. U. Sauer, "Comparison of different operation strategies for pv battery home storage systems including forecast-based operation strategies," Applied Energy, vol. 229, pp. 884-899, 2018.

[16] A. Barré, B. Deguilhem, S. Grolleau, M. Gérard, F. Suard, and D. Riu, "A review on lithium-ion battery ageing mechanisms and estimations for automotive applications," Journal of Power Sources, vol. 241, pp. 680-689, 2013.

[17] J. Li and M. A. Danzer, "Optimal charge control strategies for stationary photovoltaic battery systems," Journal of Power Sources, vol. 258, pp. 365-373, 2014. 
[18] A. Zeh and R. Witzmann, "Operational strategies for battery storage systems in low-voltage distribution grids to limit the feed-in power of roof-mounted solar power systems," Energy Procedia, vol. 46, pp. 114-123, 2014.

[19] A. Parisio, E. Rikos, and L. Glielmo, "A model predictive control approach to microgrid operation optimization," IEEE Transactions on Control Systems Technology, vol. 22, no. 5, pp. 1813-1827, 2014.

[20] F. Garcia-Torres and C. Bordons, "Optimal economical schedule of hydrogen-based microgrids with hybrid storage using model predictive control," IEEE Transactions on Industrial Electronics, vol. 62, no. 8, pp. 5195-5207, 2015.

[21] A. Santucci, A. Sorniotti, and C. Lekakou, "Power split strategies for hybrid energy storage systems for vehicular applications," Journal of Power Sources, vol. 258, pp. 395-407, 2014.

[22] E. Mayhorn, K. Kalsi, M. Elizondo, W. Zhang, S. Lu, N. Samaan, and K. Butler-Purry, "Optimal control of distributed energy resources using model predictive control," in 2012 IEEE Power and Energy Society General Meeting. IEEE, 2012, pp. 1-8.

[23] M. Petrollese, L. Valverde, D. Cocco, G. Cau, and J. Guerra, "Real-time integration of optimal generation scheduling with MPC for the energy management of a renewable hydrogen-based microgrid," Applied Energy, vol. 166, pp. 96-106, 2016.

[24] M. Khalid and A. V. Savkin, "A model predictive control approach to the problem of wind power smoothing with controlled battery storage," Renewable Energy, vol. 35, no. 7, pp. 1520-1526, 2010.

[25] J. Pahasa and I. Ngamroo, "Phevs bidirectional charging/discharging and soc control for microgrid frequency stabilization using multiple mpc," IEEE Transactions on Smart Grid, vol. 6, no. 2, pp. 526-533, 2014.

[26] SMA Solar Technology AG, "Planning guidelines - the system solution for more independence,." [Online]. Available: https://files.sma.de/dl/1353/SI-HoMan-PL-en-51.pdf

[27] J. Weniger, T. Tjaden, and V. Quaschning, "Sizing and grid integration of residential pv battery systems," in 8th International Renewable Energy Storage Conference and Exhibition (IRES 2013), Berlin, 2013.

[28] J. M. Maciejowski, Predictive control: with constraints. Pearson education, 2002.

[29] B. Kouvaritakis and M. Cannon, "Model predictive control," Switzerland: Springer International Publishing, 2016.

[30] K. S. Ng, C.-S. Moo, Y.-P. Chen, and Y.-C. Hsieh, "Enhanced coulomb counting method for estimating state-of-charge and state-of-health of lithium-ion batteries," Applied energy, vol. 86, no. 9, pp. 1506-1511, 2009.

[31] J. W. Chinneck, "Practical optimization: a gentle introduction," Systems and Computer Engineering), Carleton University, Ottawa. http://www. sce. carleton. ca/faculty/chinneck/po. html, 2006.

[32] S. Boyd and L. Vandenberghe, Convex optimization. Cambridge University Press, 2004.

[33] SMA Solar Technology AG, "Sma sunny boy datasheet,." [Online]. Available: https://www.awsolar.com.au/ wp-content/uploads/2019/06/SB15-25-DEN1915-V32print.pdf

[34] E. C. Kerrigan and J. M. Maciejowski, "Soft constraints and exact penalty functions in model predictive control," in Proc. UKACC International Conference (Control), 2000.

[35] A. Bemporad and M. Morari, "Control of systems integrating logic, dynamics, and constraints," Automatica, vol. 35, no. 3, pp. 407-427, 1999.

[36] L. Gurobi Optimization, "Gurobi optimizer reference manual," 2019. [Online]. Available: http://www.gurobi.com

[37] B. Zakeri and S. Syri, "Electrical energy storage systems: A comparative life cycle cost analysis," Renewable and sustainable energy reviews, vol. 42, pp. 569-596, 2015.

[38] Y. Censor, "Pareto optimality in multiobjective problems," Applied Mathematics and Optimization, vol. 4, no. 1, pp. 41-59, 1977.

[39] A. Del Pia, S. S. Dey, and M. Molinaro, "Mixed-integer quadratic programming is in np," Mathematical Programming, vol. 162, no. 1-2, pp. 225-240, 2017. 